\title{
Was wir vom Notfalldienst immer schon wussten...
}

\section{Rosanna Gischig, Yvonne Bogenstätter, H. Zimmermann}

Es gibt keine Interessenverbindungen.

a Der einfacheren Lesbarkeit halber wird das männliche Genus verwendet. Es sind jedoch immer beide Geschlechter angesprochen und mitgemeint.

Korrespondenz:

Prof. Dr. med. H. Zimmermann

Notfallzentrum

Inselspital

CH-3010 Bern

\section{Einleitung}

Der Notfalldienst ist heute nach wie vor ein wichtiger Bestandteil der medizinischen Grundversorgung der Bevölkerung. Diese Versorgung wird in grösseren Städten durch Spitäler mit einer Notfallstation und Hausärzte gewähr- $^{\mathrm{a}}$ leistet, während in peripheren, ländlichen Gebieten diese Aufgabe zum grössten Teil von Hausärzten übernommen wird. Sehr oft organisieren sich diese Hausärzte in der Peripherie autonom und teilen sich die Notfalldienste auf. Dies führt unter anderem auch dazu, dass die Erreichbarkeit der notfalldienstleistenden Ärztinnen und Ärzte in der Schweiz sehr unterschiedlich geregelt ist.

Je nachdem, wie viele Hausärzte in einer Region tätig sind, kann die Belastung durch den Notfalldienst beträchtlich ausfallen. Insbesondere in Winter- und Sommersportgebieten, wo die Bevölkerung während der Saison um ein vielfaches der normalen Einwohnerzahl ansteigt, ist die Belastung durch den Notfalldienst in den letzten Jahren stark angestiegen. Dies ist auf verschiedene Faktoren zurückzuführen. Zum einen ist die Ärztedichte in abgelegenen peripheren Gebieten relativ tief, d.h., die Anzahl Ärzte, die sich den Notfalldienst aufteilen müssen, ist relativ klein, und dementsprechend ist die Belastung aufgrund der permanenten Präsenz relativ hoch. Des weiteren ist die Intensität der Notfalldienste (Anzahl Patientenkontakte pro Notfalldiensttag) in diesen Gebieten äusserst unterschiedlich. Sie liegt teilweise unter einem Kontakt pro Diensttag, kann aber in Gebieten mit grossen saisonalen Schwankungen wiederum sehr beträchtlich sein. Als Folge der hohen Arbeitsbelastung der Hausärzte in der Peripherie fehlt häufig der ärztliche Nachwuchs. Bis heute fehlen jedoch weitgehend Studien, die die Arbeitssituation von Ärzten im Notfalldienst untersuchen. 2003/2004 wurden anlässlich einer Dissertation der Medizinischen Fakultät der Universität Bern die aktuelle Arbeitssituation und deren soziale Auswirkungen bei Ärzten im Notfalldienst untersucht [1].

\section{Résultats d'une étude-pilote sur}

la situation de travail actuelle des

médecins en particulier dans le

domaine du service médical de garde

Service de garde et d'urgence:

nous le savions déjà ...

Une thèse de doctorat fait le point sur la situation de travail actuelle des médecins assurant les services de garde et d'urgence ainsi que sur son impact social. L'étude a porté sur des médecins de famille du Haut-Valais et des médecins de l'Hôpital régional de Viège et du service des urgences de I'Hôpital de l'lle à Berne. Les résultats de cette étude montrent que la charge de travail dans des régions périphériques est très élevée en raison du service médical de garde et que les soins médicaux de base sont compromis notamment en raison du manque de relève. C'est pourquoi de nouvelles réglementations sont nécessaires. Dans ce contexte, le canton du Valais a lancé le $\mathbf{2}$ juillet 2007 un projet-pilote pour une durée de six mois.

\section{Methode}

\section{Stichprobe}

Es wurden 20 Interviews bei Hausärzten im Oberwallis, 20 Interviews bei Ärzten im Regionalspital Visp (seit 2004 Teil des Spitalzentrums Oberwallis) und 20 Interviews bei Ärzten im Notfallzentrum des Inselspitals Bern durchgeführt. Für die Auswahl der Ärzte wurden diese systematisch aufgrund einer selbsterstellten bzw. von den Spitälern zur Verfügung gestellten Ärzteliste angefragt und nach 20 Zusagen gestoppt. 


\section{Instrumente}

Grundlage der qualitativen Erhebung war ein Interviewleitfaden mit 22 Fragen (u. a. Arbeitssituation und Zusammenarbeit im Notfalldienst, Weiter-/Fortbildung, Befindlichkeit am Arbeitsplatz und Unterstützung, Auswirkungen auf das Sozialleben usw.).

Zwecks erhöhter Vergleichbarkeit der Resultate und zur Skalierung der qualitativen Aussagen wurde zusätzlich ein quantitativer Teil in Form eines Fragebogens erhoben. Der Fragebogen ist zweiteilig: Ein erster Teil erfasst die Arbeitszufriedenheit, die Fluktuationsabsicht sowie die Beanspruchung mittels validierter Skalen. Der zweite Teil erfasst Aspekte der Arbeitszeit wie etwa die Dauer und Intensität der Dienste, Anzahl Ruhetage und Nachtdienste usw. ${ }^{\mathrm{b}}$

\section{Erhobene Konstrukte}

\section{Arbeitszufriedenheit und Resignation}

Nach dem angewendeten Modell von Bruggemann [3] ergibt sich Arbeitszufriedenheit aus einem Soll-Ist-Vergleich zwischen Erwartungen sowie wahrgenommener Erfüllung dieser Erwartungen. Ein weiterer Faktor spielt im Zusammenhang mit Arbeitszufriedenheit ebenfalls eine wichtige Rolle, nämlich die Resignation. Dies sei an folgendem Beispiel erläutert: Ein mittlerer $\mathrm{Zu}$ friedenheitswert von «eher zufrieden» kann auf verschiedene Weise zustande kommen: Ist man «eher zufrieden» in der zuversichtlichen Erwartung auf eine mögliche Steigerung, oder ist man «eher zufrieden», weil schliesslich alles noch viel schlimmer sein könnte? Zur besseren Interpretation der Werte der Arbeitszufriedenheit wurde deshalb die Resignation miterhoben.

Insbesondere der Resignationsfaktor scheint mit körperlichen und psychosomatischen Beschwerden zu korrelieren. Aus diesem Grund haben wir emotionale Beanspruchung und $\mathrm{Pa}-$ tientenaversion in unsere Untersuchung miteinbezogen. Emotionale Beanspruchung gilt als Indikator für die Entwicklung von Burnout.

\section{Fragestellung und Auswertung}

Wir waren daran interessiert zu erfahren, ob sich die drei Berufsgruppen der Hausärzte, der Spitalärzte des Regionalspitals und der Ärzte im Notfallzentrum bezüglich Arbeitszufriedenheit, Resignation und Beanspruchung unterscheiden.

Gruppenunterschiede für Häufigkeiten zwischen den drei Berufsgruppen wurden mittels Cramers Phi getestet für dreistufige Nominalskalen. Bei fünfstufigen Skalen wurde Intervallskalenniveau angenommen und Häufigkeiten mittels Pearsons Chi-Quadrat auf Gruppenunterschiede hin überprüft. Mittelwerte wurden mittels einfaktorieller Varianzanalyse (ANOVA) auf Gruppenunterschiede hin überprüft.

\section{Resultate}

Gemäss unserer Untersuchung war die Arbeitszufriedenheit im Notfalldienst geringer als im regulären Dienst, wobei dieser Unterschied statistisch signifikant war. Im regulären Dienst war die Resignation weniger stark ausgeprägt, wobei dieser Unterschied auch statistisch signifikant war. Im weiteren zeigte die Untersuchung, dass im Notfalldienst eine abnehmende Arbeitszufriedenheit mit zunehmender emotionaler Erschöpfung, zunehmender Patientenaversion und zunehmender Fluktuationsabsicht einhergeht. Die Korrelation ist signifikant. Gemäss Beantwortung der Fragen bezüglich der Beanspruchungen bei der Arbeit befinden sich 8 von 56 Ärzten bezüglich emotionaler Erschöpfung im kritischen Bereich (Abb. 1).

Interessant war in diesem Zusammenhang, dass beim Vergleich der emotionalen Erschöpfung zwischen den drei untersuchten Gruppen keine signifikanten Gruppenunterschiede festzustellen waren. Gemäss der Beantwortung der Fragen zur Patientenaversion befinden sich 7 von 56 Ärzten im kritischen Bereich (Abb. 2).

Ebenso wie bei der emotionalen Erschöpfung gab es auch bei der Patientenaversion keine signifikanten Unterschiede zwischen den drei Gruppen. Ein weiterer Aspekt war die persönliche Einflussmöglichkeit auf die Gestaltung der Arbeitszeit im Notfalldienst. Diese werden von 40 von 56 Ärzten als gering oder mittel eingestuft. Diese mangelnde Gestaltungsmöglichkeit ist mit ein Grund für eine tiefere Arbeitszufriedenheit im Notfalldienst.

Gemeinsam ist allen drei untersuchten Gruppen das Auftreten von belastenden Situationen im Notfalldienst. Vor allem jüngere Ärzte sprechen von Hilflosigkeit, Insuffizienzgefühlen und daraus folgender Demotivation. Unterschiedlich ist der Umgang mit solchen Situationen. Verschiedene Bewältigungsstrategien wurden genannt. In den Interviews kommt klar zum Vorschein, dass die Ärzte aller drei Gruppen soziale Unterstützung beanspruchen, entweder am Arbeitsplatz und/oder aus dem privaten Umfeld. Die Resultate dieser Untersuchung zeigen zudem, dass die Arbeitsbelastung durch den Notfalldienst bei den Hausärzten regional sehr unterschiedlich ausfällt. Insbesondere in peripheren Regionen mit geringer Ärztedichte, aber saisonal stark schwankenden Patientenzahlen, kann die 
Abbildung 1

Emotionale Erschöpfung

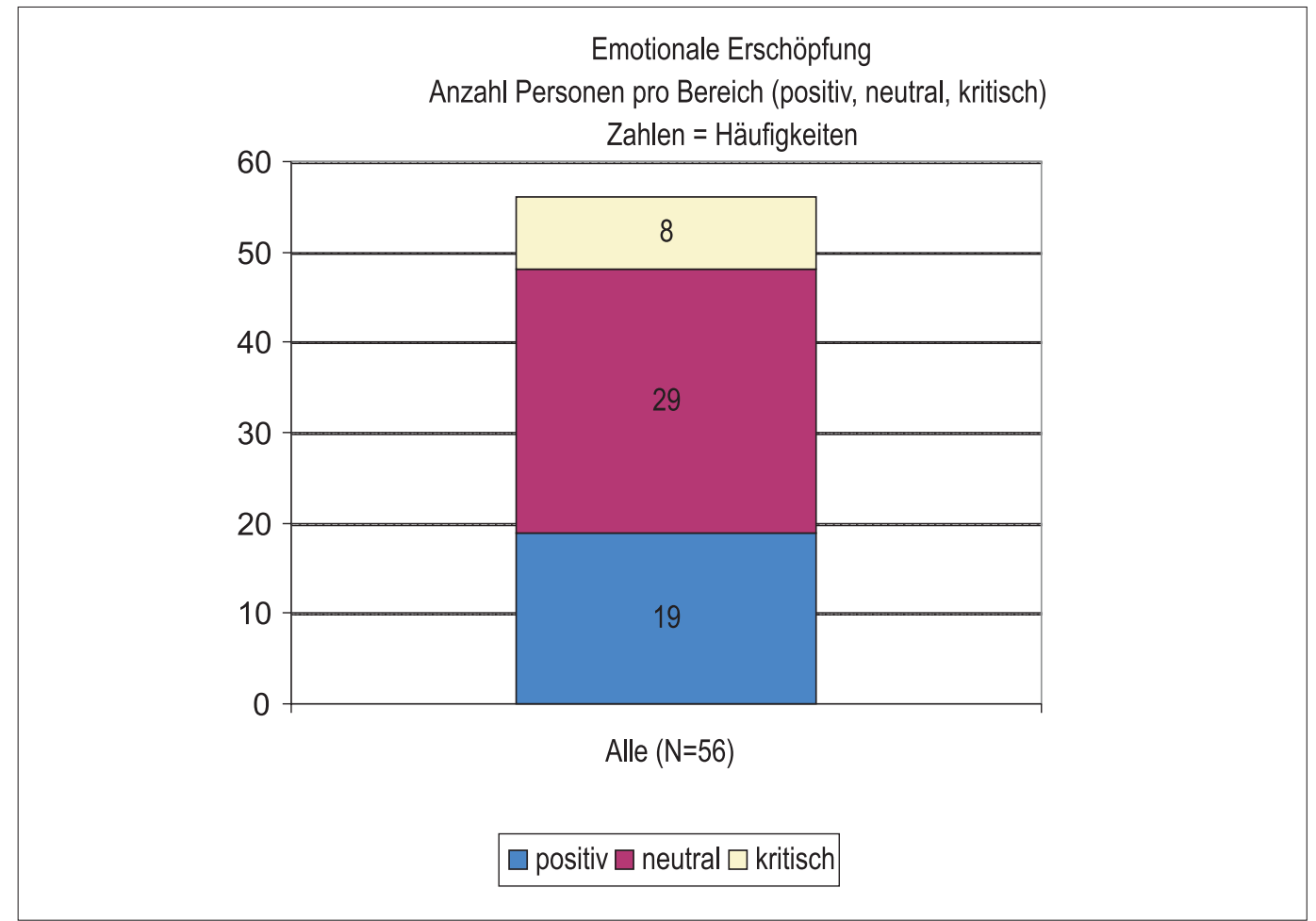

Positiv = keine emotionale Erschöpfung vorhanden; neutral = unauffälliger Bereich der emotionalen Erschöpfung; kritisch = emotionale Erschöpfung im kritischen Bereich.

\section{Abbildung 2}

Patientenaversion.

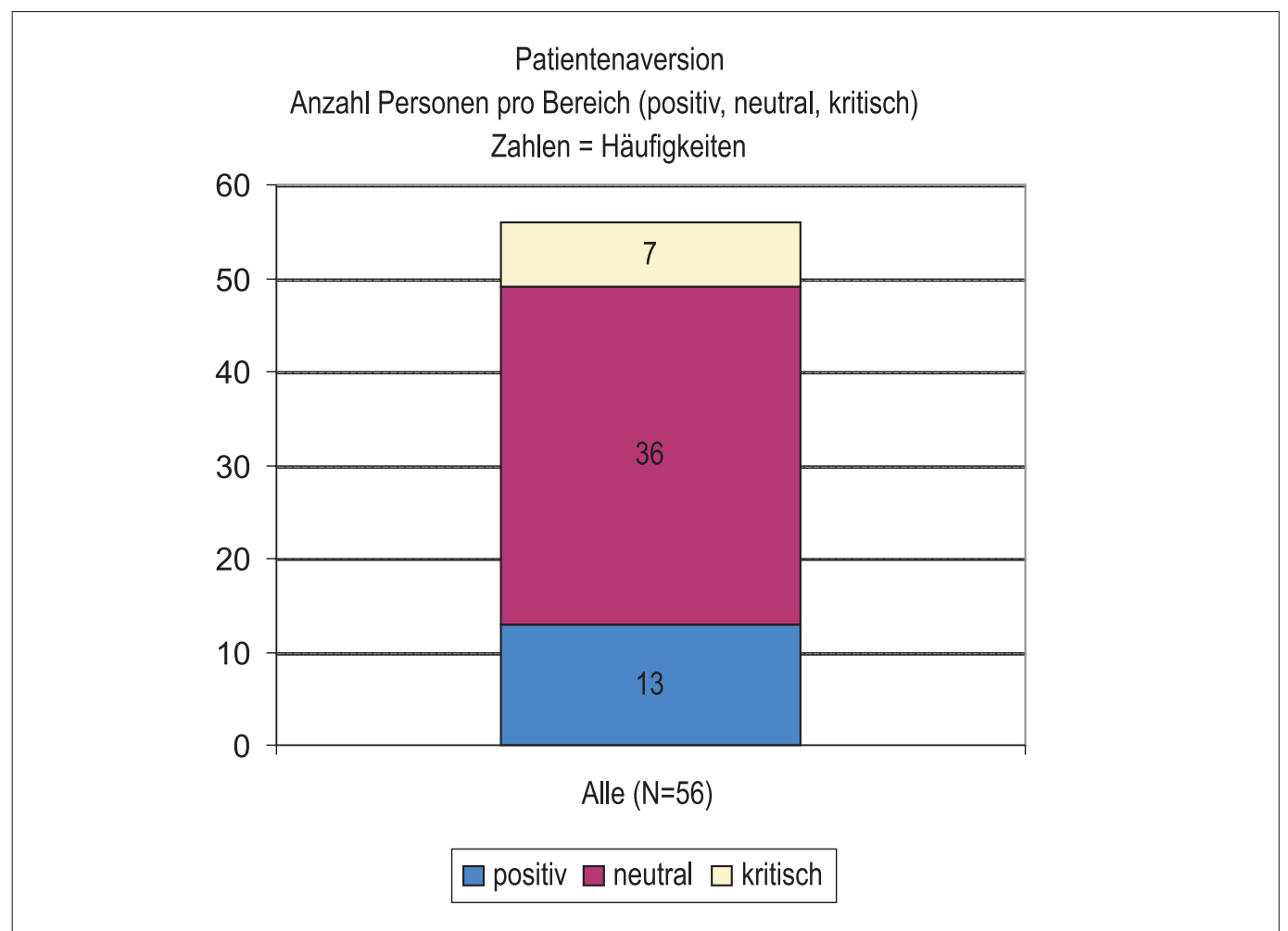

Positiv = keine Patientenaversion vorhanden; neutral = unauffälliger Bereich der Patientenaversion; kritisch $=$ Patientenaversion im bedenklichen Ausmass. 
Belastung sehr hoch ausfallen. Die Situation in der Peripherie wird zusätzlich durch die Tatsache verschärft, dass trotz intensiver Suche keine zusätzlichen Hausärzte bzw. Nachfolger gefunden werden können. Verständlicherweise sehen diese Hausärzte die Gewährleistung der medizinischen Grundversorgung der Bevölkerung in näherer Zukunft gefährdet. 11 der 20 befragten Hausärzte gaben an, dass sie sich durch die Arbeit im Notfalldienst in ihrem Sozialleben beeinträchtigt fühlen. Zusätzlich haben sich diese Hausärzte über eine mangelnde Honorierung der grossen Präsenzzeit beklagt sowie eine ständig steigende Erwartung der Bevölkerung bezüglich Verfügbarkeit der Hausärzte. Im weiteren sind die Hausärzte häufig auf sich selbst gestellt. Um so wichtiger ist ein gutes Unterstützungssystem, das wahrscheinlich einen wesentlichen Beitrag zur Verhinderung von Burnout leisten kann. Zudem beklagen sich die Hausärzte in der Peripherie über mangelnde Zeit für die Fortbildungen. Es fehlt aufgrund der intensiven Dienste und aufgrund der Distanz die Zeit, Fortbildungen zu besuchen. Eine weitere Problematik besteht in der Zusammenarbeit zwischen Hausärzten und Spitalärzten. Sowohl von seiten der Hausärzte als auch von seiten der Spitalärzte werden mangelnde Information und Probleme in der Kontinuität der Patientenbetreuung als Kritikpunkte angegeben.

\section{Diskussion}

Was seit längerer Zeit regelmässig diskutiert wird, hat sich auch in der Untersuchung anlässlich dieser Dissertation gezeigt und konnte mit Fakten und Zahlen belegt werden. Dieses alte Modell der Notfalldienstorganisation hat wahrscheinlich bald ausgedient und ist so auch nicht mehr lebbar. Neue Modelle der Notfalldienstregelung müssen gefunden werden. In der Schweiz gibt es diesbezüglich keine einheitliche Lösung. Je nach Region und Bedürfnissen der Bevölkerung müssen andere Lösungen gefunden werden.
Seit dem 2. Juli 2007 läuft im Wallis ein zunächst sechsmonatiges Pilotprojekt [5]. Der Walliser Ärzteverband, die Kantonale Walliser Rettungsorganisation und die Dienststelle für Gesundheitswesen schaffen mit Unterstützung des Kantons eine zentrale Leitstelle für den ärztlichen Bereitschaftsdienst. Patienten sollen künftig während der Nacht und am Wochenende über eine kantonsweit einheitliche Telefonnummer Auskünfte erhalten. Mit einer Triage bereits am Telefon sollen künftig unnötige Konsultationen und Hospitalisationen vermieden werden. Falls eine Arztkonsultation notwendig ist, wird diese auch durch den entsprechenden diensthabenden Arzt in der Region gewährleistet. Ziel ist es, dass unter anderem auch der Hausarztberuf in peripheren Regionen wieder attraktiver wird. Bleibt nur zu hoffen, dass sich dieses Pilotprojekt bewährt und die Finanzierung eines solchen Systems auch für die Zukunft gewährleistet ist. Wer möchte schon seine Sportferien in einer Region verbringen, die ärztlich unterversorgt ist? Ist doch der Tourismus ein wichtiger Wirtschaftszweig in den Randregionen der Schweiz.

An dieser Stelle soll allen beteiligten Ärzten für ihre Teilnahme an der Untersuchung gedankt werden.

\section{Literatur}

1 Gischig R. Aktuelle Arbeitssituation und deren soziale Auswirkungen bei Ärzten unter besonderer Berücksichtigung des Notfalldienstes. Dissertation. Universität Bern; 2005.

2 Baillod J, Semmer N. Fluktuation und Berufsverläufe bei Computerfachleuten. Zeitschrift für Arbeits- und Organisationspsychologie. 1994;38: 152-63.

3 Bruggemann A, Groskurth P, Ulich E. Arbeitszufriedenheit. Bern: Huber; 1975.

4 Hacker W, Reinhold S. Beanspruchungsscreening bei Humandienstleistungen (BHD-System). Frankfurt a.M.: Swets Test Services; 1999.

5 Hier werden Sie verarztet. Walliser Bote, 20. Juni 2007, S. 3. 